Coher ence- $t r$ ansfer $r$ ot ary echoes produced by doubl e i rradi at i on i $n$ a three- I evel NMR system

\begin{tabular}{|l|l|}
\hline 著者 & Hat anaka H roshi, Hashi Tsuneo \\
\hline $\begin{array}{l}\text { j our nal or } \\
\text { publ i cat i on t i t l e }\end{array}$ & Physi cal Revi ew B \\
\hline vol une & 21 \\
\hline number & 7 \\
\hline page r ange & $2677-2680$ \\
\hline year & 1980 -01-01 \\
\hline URL & ht t p: //hdl . handl e. net /2297/7565 \\
\hline
\end{tabular}




\title{
Coherence-transfer rotary echoes produced by double irradiation in a three-level NMR system
}

\author{
Hiroshi Hatanaka \\ Department of Phisics. Faculty of Education. Kanazawa Universit!. Kanazawa 920. Japan \\ Tsuneo Hashi \\ Department of Phisics. Faculty of Science. Kioto Universit!. K!roto 606. Japan
}

(Received 30 July 1979)

\begin{abstract}
A new type of spin echoes associated with periodic transfer of coherence has been observed in a three-level NMR system under irradiation with two $\mathrm{rf}$ fields which are exactly at resonance to the two single-quantum transitions. The echoes are observed for the two-quantum coherences as well as for the single-quantum ones. The echo-envelope decay is considerably lengthened by the double irradiation because of the quenching of the magnetic dipolar interaction.
\end{abstract}

There has been considerable recent interest in coherent transient phenomena in a three-level system both in NMR and quantum optics. Besides the wellknown two-quantum (two-photon) coherent transient phenomena where the intermediate state is off resonance, ${ }^{1}$ there are phenomena peculiar to the threelevel system where the intermediate state is resonant. $^{2-4}$ In this paper, we report a new type of spin echo belonging to the latter category. This echo is associated with the transfer of coherences produced by double irradiation with two rf fields which are exactly at resonance with the two single-quantum transitions. The feasibility of this type of echo formation is implicitly involved in a previous work ${ }^{2}$ on the periodic transfer of coherences in a multilevel NMR system. However, in practice, when single irradiation is used, the decay time of the coherence is too short to observe this type of echo. In the case of double irradiation, the decay time is considerably lengthened, as will be shown later, because of the quenching of the magnetic dipolar interaction. We have observed echoes associated with two-quantum coherences as well as those with single-quantum ones. The mechanism of the echo formation is in some respects similar to that of the rotary echo. ${ }^{5}$ However, the present echoes are quite different from ordinary rotary echoes and even from two-quantum rotary echoes in a three-level system. ${ }^{6}$ The mutual transfer of coherences among three-level pairs is essential in echo formation, and the system must be prepared by an appropriate initial condition. This type of echo may be observed at optical frequencies.

We first derive the equation of motion representing periodic transfer of coherences produced by the double irradiation in a three-level system. Previous theoretical studies of the two-quantum (two-photon) coherent transient phenomena in a three-level system with resonant intermediate state $e^{4,7}$ are not applicable or not suitable for the present work. Consider an
NMR system consisting of the levels $a, b$, and $c$ as shown in Fig. 1(a), where level spacings are unequal and magnetic dipolar transitions $(M 1)$ between levels $a$ and $b(a \multimap b)$ and $b$ and $c(b \rightarrow c)$ are allowed, but that between $a$ and $c(a-c)$ is forbidden. Suppose that two rf magnetic fields (i) $H_{x 1}(t)=H_{1} \cos \left(\omega_{1} t\right)$ and (ii) $H_{x 2}(t)=H_{2} \cos \left(\omega_{2} t\right)$ which are exactly at resonance with the transitions $a \rightarrow b$ and $b \rightarrow c$, respectively, are applied to the spin system along the $x$ axis, which is perpendicular to the quantization $(z)$ axis of the three-level system.

The Hamiltonian $\mathcal{H}$ of the system can be written

$$
\mathcal{H C}=\mathcal{K}_{0}-\gamma \hbar\left[H_{x 1}(t)+H_{x 2}(t)\right] \sum_{j} I_{x j}
$$

where $\mathcal{J}_{0}$ is an unperturbed Hamiltonian describing the three-level system, $I_{j}$ the $j$ th nuclear spin, and $\gamma$ (assumed to be positive) the gyromagnetic ratio. We assume that $\left|\omega_{1}-\omega_{2}\right| \gg>\beta_{a b}, \beta_{b c}$, where $2 \beta_{a b}$ $=\gamma H_{1}\left\langle a\left|I_{x}\right| b\right\rangle$ and $2 \beta_{b c}=\gamma H_{2}\left\langle b\left|I_{x}\right| c\right\rangle$ are nutation frequencies associated with single-quantum transitions $a \multimap b$ and $b \rightarrow c$, respectively. Using the rotating-wave approximation and neglecting rapidly oscillating terms, we obtain the equation of motion of the density matrix $\rho$ in the interaction representation, eliminating the effect of $\mathcal{K}_{0}$, as

$$
\dot{\rho}=-i\left(\rho, \beta_{a b} \sum_{j} Q_{x j}(a b)+\beta_{b c} \sum_{j} Q_{x j}(b c)\right) \text {, }
$$

where the $Q$ 's are operators ${ }^{8}$ defined by using eigenfunctions $|m\rangle_{j}$ and $|n\rangle_{j}$ of $I_{z j}$ as follows:

$$
\begin{aligned}
& Q_{x j}(m n)=|m\rangle_{j j}\langle n|+| n\rangle_{j j}\langle m|, \\
& Q_{y j}(m n)=-i\left(|m\rangle_{j j}\langle n|-| n\rangle_{j j}\langle m|\right), \\
& Q_{z j}(m n)=|m\rangle_{j j}\langle m|-| n\rangle_{j j}\langle n| .
\end{aligned}
$$

The equations of motion of the coherences defined 


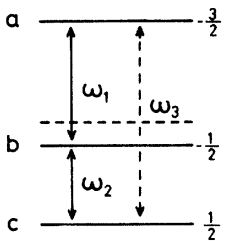

(a)

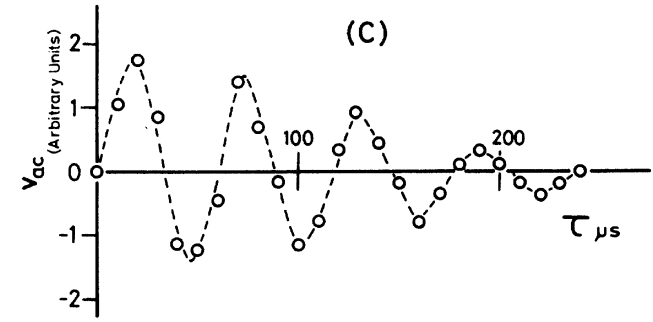

FIG. 1. (a) Energy-level diagram of the three-level system. (b) Experimental operations to observe oscillatory behavior of the two-quantum coherence $v_{a c}$ under the irradiation with two resonant $\mathrm{rf}$ fields. The spin-locking at $\omega_{1}$ is to provide the initial condition $u_{a b} \neq 0, u_{b c}=v_{a c}=0$. The $180^{\circ}$ pulse at $\omega_{2}$ is the probe pulse to detect $v_{a c}$. (c) The oscillation of $v_{a c}$ as a function of $\tau$. The dashed line is experimental.

by

$$
u_{m n}=\rho_{m n}+\rho_{n m}=\operatorname{Tr}\left(\rho \sum_{j} Q_{x j}(n m)\right)
$$

and

$$
v_{m n}=-i\left(\rho_{m n}-\rho_{n m}\right)=\operatorname{Tr}\left(\rho \sum_{j} Q_{y j}(n m)\right),
$$

and those of the population differences

$$
w_{m n}=\rho_{n n}-\rho_{m m}=\operatorname{Tr}\left(\rho \sum_{j} Q_{z j}(n m)\right)
$$

can be derived from Eq. (2). These equations can be divided into two independent sets, which is possible only when the rf fields (i) and (ii) are exactly at resonance. The first set is

$$
\begin{aligned}
& \dot{u}_{a b}=\beta_{b c} v_{a c}, \\
& \dot{u}_{b c}=-\beta_{a b} v_{a c}, \\
& \dot{v}_{a c}=\beta_{a b} u_{b c}-\beta_{b c} u_{a b} .
\end{aligned}
$$

This is the basic equation in the following discussion. It is to be noted that Eq. (4) contains only the coherences $u_{a b}, u_{b c}$, and $v_{a c}$ and no population differences $w_{i j}(i, j=a, b, c)$. The second set, which is more complicated, contains coherences $v_{a b}, v_{b c}, u_{a c}$ and population differences $w_{i j}$. If one starts from thermal equilibrium and just applies two rf fields which are exactly at resonance with the single-quantum transi- tions, only the phenomena governed by the second set of equations can be observed. However, if one provides the initial condition such that at least one of the coherences $u_{a b}, u_{b c}$, and $v_{a c}$ exists at $t=0$, one can observe the behavior governed by Eq. (4). If one introduces a state vector $\overrightarrow{\mathrm{r}}=\left(u_{a b}, u_{b c}, v_{a c}\right)$ and an effective angular velocity vector $\overrightarrow{\mathrm{q}}=\left(\beta_{a b}, \beta_{b c}, 0\right)$ in a virtual three-dimensional space, Eq. (4) can be rewritten as a torque equation $\dot{\vec{r}}=\vec{q} \times \vec{r}$. The vector model based on this equation shows that the periodic transfer occurs among the two-quantum coherence $v_{a c}$ and the single-quantum ones $u_{a b}$ and $u_{b c}$ with frequency $\alpha=\left(\beta_{a b}^{2}+\beta_{b c}^{2}\right)^{1 / 2}$. Note that half the nutation frequencies contribute to $\alpha$. The explicit expression of the solution of Eq. (4) for a simple initial condition $\vec{r}(0)=\left(u_{a b}(0), 0,0\right)$ is

$$
\begin{aligned}
& u_{a b}(t)=u_{a b}(0)\left(\beta_{a b}^{2}+\beta_{b c}^{2} \cos \alpha t\right) / \alpha^{2}, \\
& u_{b c}(t)=u_{a b}(0) \beta_{a b} \beta_{b c}(1-\cos \alpha t) / \alpha^{2}, \\
& v_{a c}(t)=-u_{a b}(0) \beta_{b c}(\sin \alpha t) / \alpha .
\end{aligned}
$$

The experiment was carried out on ${ }^{27} \mathrm{Al}$ nuclei $\left(I=\frac{5}{2}\right.$ ) in $\mathrm{Al}_{2} \mathrm{O}_{3}$ at $77 \mathrm{~K}$. A static magnetic field $H_{0}$ was applied along the principal axis ( $z$ axis) of the electric-field gradient (efg) tensor (asymmetry parameter $\eta=0$ ) at $\mathrm{Al}$ nuclei. The levels with $I_{z}=-\frac{3}{2}$, $-\frac{1}{2}$, and $\frac{1}{2}$ were used as $a, b$, and $c$, respectively, and $\omega_{1} / 2 \pi=10 \mathrm{MHz}$ and $\omega_{2} / 2 \pi=9.64 \mathrm{MHz}$. The free induction decay (FID) signals which are in phase with the applied rf fields (i) and (ii) were selectively detected by a phase-sensitive detector. The observed phenomena occur in a time much shorter than the spin-lattice relaxation time $T_{1}$.

In order to observe the oscillatory behavior of the two-quantum coherence $v_{a c}(t)$, we used the experimental sequence shown in Fig. 1(b). The initial condition $\vec{r}(0)=\left(u_{a b}(0), 0,0\right)$ is achieved by spin locking with the rf field (i), and then the rf field (ii) is turned on. The system is simultaneously irradiated by the two resonant rf fields for a time $\tau$. Since the two-quantum coherence $v_{a c}$ is not accompanied by an oscillating magnetization, it cannot be detected directly by a pick-up coil. As in the previous expeirments, ${ }^{2}$ a probe pulse $\left(180^{\circ}\right.$ pulse at $\left.\omega_{2}\right)$ is used to detect it as the amplitude of FID signal at $\omega_{1}$. Figure 1(c) shows experimental results. The frequency of the oscillation is in agreement with the calculated value of $\alpha / 2 \pi=\left(\beta_{a b}^{2}+\beta_{b c}^{2}\right)^{1 / 2} / 2 \pi=17 \mathrm{kHz}\left(H_{1}=13\right.$ Oe and $\left.H_{2}=16 \mathrm{Oe}\right)$.

Similar oscillatory behavior was observed for the single-quantum coherences $u_{a b}$ and $u_{b c}$. The frequencies and the relative phases of the oscillations are in good agreement with the predictions of Eq. (5). The oscillations of the single-quantum coherences were observed by Stoll et al. ${ }^{9}$ in connection with the study of spinor character of the spin system $(I=1)$. 
The origin of the damping of the oscillations of the coherences $v_{a c}, u_{a b}$, and $u_{b c}$ will be discussed later.

The new type of spin echo is associated with these oscillations. When a spatial inhomogeneity is introduced in $H_{1}$ and/or $H_{2}$, the system can be divided into subsystems for which the oscillation frequencies $\alpha$ 's and the directions of the vectors $\vec{q}$ are different. This causes the damping of the oscillations of the macroscopic coherences averaged over the sample. However, if all the vectors $\vec{q}$ are inverted, this damping should be recovered and the echoes be formed in a manner similar to that of rotary echoes. This pictorial description can be confirmed analytically by using Eq. (4). The inversion of the vector $\vec{q}$ is accomplished by simultaneous rf phase shifts by $180^{\circ}$ of two rf fields (i) and (ii) as verified by the theory, namely, we obtain the same type of equation as Eq. (4) where the signs of $\beta$ 's are changed, when the phases of the two rf fields are shifted by $180^{\circ}$.

The echoes associated with two-quantum coherence $v_{a c}$ were observed by using the operation represented as (A) in Fig. 2. After the simultaneous phase shifts at $\tau=\tau_{0}(=110 \mu \mathrm{s})$ the damping of the oscillation due to the inhomogeneities is recovered and the echo centered at $\tau=2 \tau_{0}$ appears as shown in the upper part of the figure.

The echoes were observed for any of the coherences $u_{a b}, u_{b c}$, and $v_{a c}$. An example for $u_{a b}$ is also shown in Fig. 2 together with the operation [represented as (B)].

We call these echoes "coherence transfer rotary echoes (CTRE)". Although the mechanism of echo formation in the vector model is similar to that of the rotary echo, these are new phenomena arising from the periodic transfer of coherence among three level pairs. Recently, Maudsley et al. reported echoes similarly named, coherence transfer echoes. ${ }^{10}$ In their experiments the transfer of coherence is used to change the level pairs where the dephasing and rephasing occur. In the present echoes the dephasing and rephasing take place while the transfer of coherences occurs continuously under the irradiation of the rf fields.

As mentioned above, the CTRE should be obtained, in principle, by a single irradiation. Calculations show that if one applies only one rf field, say

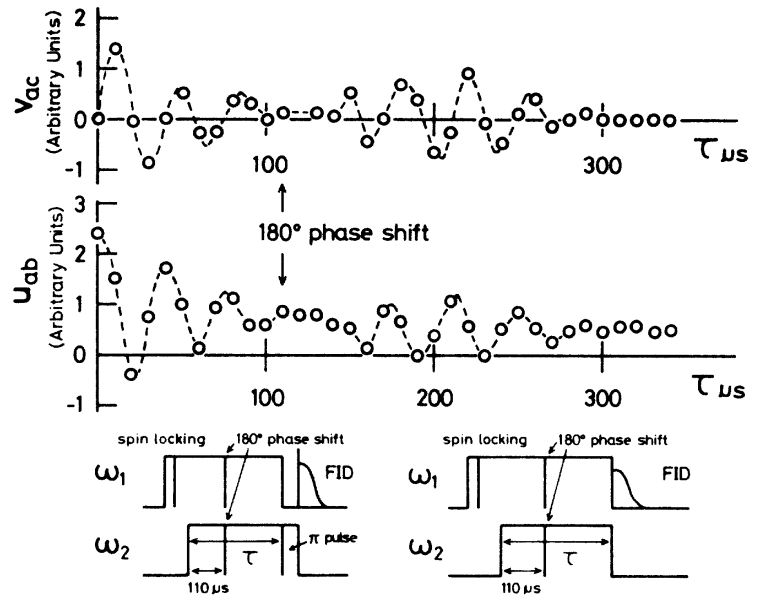

(A)

(B)

FIG. 2. Coherence transfer rotary echoes associated with two-quantum coherence $v_{a c}$ and single-quantum coherence $u_{a b}$ obtained by the operations (A) and (B), respectively. The simultaneous $180^{\circ}$ phase shifts are made at $\tau=110 \mu \mathrm{s}$ and the echo peaks appear at $\tau=220 \mu \mathrm{s}$. The $90^{\circ}$ phase difference is observed in the oscillations of $v_{a c}$ and $u_{a b}$. The dashed lines are experimental.

(ii), in the presence of $u_{a b}(0)$ and shifts the phase by $180^{\circ}$ at $\tau=\tau_{0}$, one can expect CTRE at $\tau=2 \tau_{0}$.

However, as will be described later, the decay time of the oscillation of the coherence is so short that it is in practice difficult to observe CTRE.

The observed echo-envelope decays agreed well with the decay curves of the oscillations obtained in the absence of the field inhomogeneities of $H_{1}$ and $H_{2}$. These decays are due to the magnetic dipolar interaction $\mathcal{H}_{d}$ between $\mathrm{Al}$ spins. Considering $\mathcal{H}_{d}$ as a perturbation and assuming Gaussian decay proportional to $\exp \left(-t^{2} / 2 T_{2}^{2}\right)$, we obtain

$$
T_{2}^{-1}=\left\{\frac{-\operatorname{Tr}\left\{\left[\sum_{j} Q_{x j}(a b), \mathcal{K}_{d}^{*}\right]^{2}\right\}}{\hbar^{2} \operatorname{Tr}\left(\sum_{j} Q_{x j}(a b)\right]^{2}}\right\}^{1 / 2},
$$

where $\mathcal{H C}_{d}^{*}$ represents the time-independent part of

$$
\exp \left(-i \alpha \sum_{j} Q_{x j}(c b) t\right) \exp \left(i \theta \sum_{j} Q_{y j}(c a)\right) 3 c_{d}^{0} \exp \left(-i \theta \sum_{j} Q_{y j}(c a)\right) \exp \left(i \alpha \sum_{j} Q_{x j}(c b) t\right)
$$

$\mathcal{K}_{d}^{0}$ is the secular part of $\mathcal{K}_{d}$ given by

$$
\mathcal{K}_{d}^{0}=\frac{1}{2} \sum_{j, k}\left\{I_{z j} I_{z k}-\frac{1}{8} \sum_{n}\left\langle n\left|I_{+}\right| n-1\right\rangle\left[Q_{x j}(n, n-1) Q_{x k}(n, n-1)+Q_{y j}(n, n-1) Q_{y k}(n, n-1)\right]\right) D_{j k}
$$

and $\cos 2 \theta \equiv p=\left(\beta_{b c}^{2}-\beta_{a b}^{2}\right) / \alpha^{2}$. It can be shown that the decay time $T_{2}$ increases with increasing $|p|$ and has a maximum near $p=0$ and a minimum near $|p|=1$. The geometric factor $\sum_{j(\neq k)} D_{j k}^{2}$ can be es- 
timated from the observed decay time $(80 \mu \mathrm{s})$ of FID for the single-quantum transition $(a-b)$ with an aid of the expression of the second moment. ${ }^{11}$ The maximum value of $T_{2}$ estimated is about $350 \mu \mathrm{s}$, which is much longer than the decay time of the FID for the single-quantum transitions. The observed decay time when $\beta_{a b} \sim \beta_{b c}$ was about $400 \mu \mathrm{s}$. The lengthening is due to the quenching of the magnetic dipolar interaction by the double irradiation. This is a kind of line narrowing in a three-level system. The theoretical and experimental values of the minimum decay time when $\beta_{a b}=0$ were 70 and $73 \mu \mathrm{s}$, respectively.

Steady-state values of $v_{a c}, u_{a b}$, and $u_{b c}$ obtained from Eq. (5) with damping factor are $0, \beta_{a b}^{2} u_{a b}(0) /$ $\alpha^{2}$, and $\beta_{a b} \beta_{b c} u_{a b}(0) / \alpha^{2}$, respectively. The experimental values were in agreement with the theoretical ones. The latter two values can also be obtained by using the spin-temperature hypothesis. ${ }^{5}$ In the steady state, two single-quantum transitions $a-b$ and $b \rightarrow c$ are simultaneously spin locked by the rf fields (i) and (ii), respectively.

We also carried out experiments under a different initial condition: $\vec{r}(0)=\left(0,0, v_{a c}(0)\right)$, where the vec- tor $\vec{r}$ is perpendicular to the vector $\vec{q}$. This initial condition was achieved by applying a $90^{\circ}$ pulse with frequency $\omega_{1}$ followed by a $180^{\circ}$ pulse with frequency $\omega_{2}$. Experimental results are quite similar to those in the above except that all the coherences $v_{a c}, u_{a b}$, and $u_{b c}$ decay to zero after the oscillations.

Finally we propose a possible experiment using a triple irradiation. Now we assume that the transition $a \rightarrow c$ is $M 1$ allowed and consider that the third of field $H_{3} \sin \omega_{3} t$ which is exactly at resonance to the transition $a \rightarrow c$ is applied along the $x$ axis in addition to the rf fields (i) and (ii). The calculation shows that the behavior of the spin system can be described by a torque equation of the same form with vector $\overrightarrow{\mathrm{q}}=\left(\beta_{a b}, \beta_{b c}, \beta_{a c}\right)$, where $\beta_{a c}=\frac{1}{2} \gamma H_{3}\left\langle a\left|I_{x}\right| c\right\rangle$. The periodic transfer of coherence among $u_{a b}, u_{b c}$, and $v_{a c}$ is expected to occur at the frequency $\left(\beta_{a b}^{2}+\beta_{b c}^{2}\right.$ $\left.+\beta_{a c}^{2}\right)^{1 / 2}$, and CTRE's are expected in a similar way when the phases of all rf fields are simultaneously shifted by $180^{\circ}$. A more effective quenching of the magnetic dipolar interaction could be expected in this case.
${ }^{1}$ See for example, H. Hatanaka and T. Hashi, J. Phys. Soc. Jpn. 39, 1139 (1975); A. Pines, S. Vega, D. J. Ruben, T. W. Shattuck, and D. E. Wemmer, Lecture presented at the Ampere Summer School Pula, Yugoslavia, September 1976 (unpublished); D. Grischkowsky and M. M. T. Loy, Phys. Rev. A 12, 2514 (1975); A. Flusberg, T. Mossberg, R. Kachru, and S. R. Hartmann, Phys. Rev. Lett. 41, 305 (1978).

${ }^{2}$ H. Hatanaka, T. Terao, and T. Hashi, J. Phys. Soc. Jpn. 39, 835 (1975).

${ }^{3}$ T. Mossberg, A. Flusberg, R. Kachru, and S. R. Hartmann, Phys. Rev. Lett. 39, 1523 (1977)

${ }^{4}$ M. Aihara and H. Inaba, J. Phys. A $\underline{6}, 1709,1725$ (1973).

${ }^{5}$ A. Abragam, The Principle of Nuclear Magnetism (Clarendon, Oxford, 1961).
${ }^{6}$ H. Hatanaka, T. Ozawa, and T. Hashi, J. Phys. Soc. Jpn. 42, 2069 (1977).

${ }^{7}$ R. G. Brewer and E. L. Hahn, Phys. Rev. A $\underline{11}, 1641$ (1975).

${ }^{8} \mathrm{H}$. Hatanaka, thesis (Kyoto University, 1977)

(unpublished). Essentially the same operators are used independently by A. Wokaun and R. R. Ernst, J. Chem. Phys. 67, 1752 (1977); and D. Suwelack, M. Mehring, and A. Pines, Phys. Rev. B $\underline{19}, 238$ (1979).

${ }^{9}$ M. E. Stoll, E. K. Wolff, and M. Mehring, Phys. Rev. A $\underline{17}$, 1561 (1978).

${ }^{10}$ A. A. Maudsley, A: Wokaun, and R. R. Ernst, Chem. Phys. Lett. 55, 9 (1978).

${ }^{11}$ D. G. Hughes and P. A. Spencer, Phys. Lett. A $\underline{71}, 123$ (1979); H. Hatanaka and T. Hashi (unpublished). 